Matthew R. Ginsberg, MD

Neurodevelopmental Science Center, Akron Children's

Hospital, Akron, OH; Assistant Professor of Pediatrics,

Northeast Ohio Medical University, Rootstown, $\mathrm{OH}$
John A. Morren, MD

Program Director, Neuromuscular Medicine Fellowship, Neurological Institute, Cleveland Clinic; Clinical Assistant Professor, Cleveland Clinic Lerner College of Medicine of Case Western Reserve University, Cleveland, $\mathrm{OH}$
Kerry Levin, MD

Chair, Department of Neurology, and Director, Neuromuscular

Center, Neurological Institute, Cleveland Clinic; Professor,

Cleveland Clinic Lerner College of Medicine of Case Western

Reserve University, Cleveland, $\mathrm{OH}$

\title{
Using and interpreting electrodiagnostic tests
}

\section{ABSTRACT}

Electrodiagnostic testing, consisting of nerve conduction studies and needle electrode examination, serves as an extension of a neurologic examination for evaluating a variety of focal and generalized neuromuscular conditions. By providing important clues on location, chronicity, severity, and pathophysiology, it can help to establish a diagnosis, evaluate the need for surgery, and assess patients who do not improve as expected after surgery.

\section{KEY POINTS}

Electrodiagnostic testing helps to precisely locate disease processes affecting the peripheral nervous system (including peripheral nerves, neuromuscular junctions, and muscles) and has limited use in the evaluation of central nervous system disorders.

Electrodiagnostic studies can help establish if a patient is likely to have a muscle disease, a disorder of neuromuscular junction transmission, axon loss, or a demyelinating disease.

Electrodiagnostic testing should be done by physicians who have appropriate training in it, as there are potential pitfalls in performing and interpreting the studies.
The authors report no relevant financial relationships which, in the context of their contributions, could be perceived as a potential conflict of interest.
Lectrodiagnostic testing (traditionally C but less accurately called electromyography) consists of 2 distinct but related procedures typically performed together to interrogate the peripheral nervous system: nerve conduction studies and needle electrode examination.

This article reviews common indications for these tests, their limitations, and how to interpret the results, focusing on how they may best contribute to patient evaluation.

\section{NERVE CONDUCTION STUDIES}

Nerve conduction studies involve stimulating motor, sensory, or mixed nerves through the skin with a small pulse of electrical current (Figure 1). Recording electrodes, placed on the skin over nerves and muscles innervated by the stimulated nerve trunk, capture electrical responses generated by the stimulation. Multiple nerves may be stimulated in each affected limb or region, as determined by patient symptoms.

Sensory nerve conduction studies record the response along nerve fibers to electrical stimulation of the nerve trunk at some distance from the recording electrodes, whereas motor nerve conduction studies record the response of a muscle to electrical stimulation of a nerve trunk that innervates that muscle.

Values measured include amplitude and morphology of response and velocity or latency of conduction along the stimulated path. "Late" responses, including the $\mathrm{F}$ wave and $\mathrm{H}$ reflex, measure the integrity of proximal portions of a nerve and corresponding nerve roots.

The following disease processes are generally associated with characteristic electrodiagnostic findings, illustrated in Figure 2: 


\section{Nerve conduction studies: Principles}
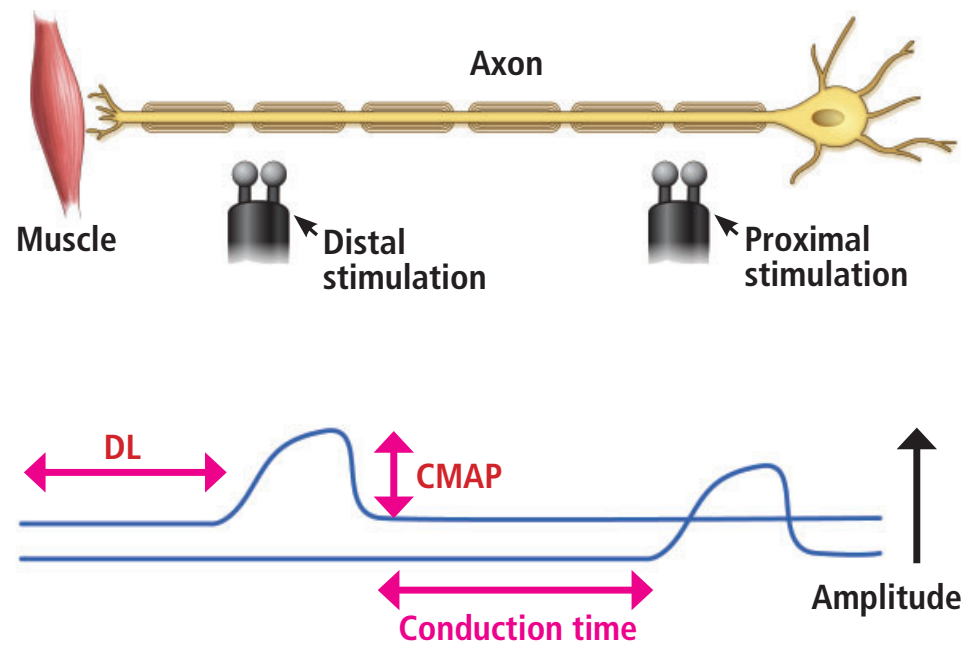

Time
Figure 1.

Electrodiag-
nostic testing
consists of
nerve
conduction
studies
and needle
electrode
examination

Demyelinating diseases cause slow conduction velocities, prolonged distal latencies, conduction blocks, dispersion of the motor response waveform, and prolonged late responses.

Axon loss ("axonal pathology") does not significantly exhibit these features, but causes reduced amplitude of responses.

Acquired focal or segmental demyelination characteristically exhibits conduction block, ie, a significant reduction in motor response amplitude at proximal compared with distal stimulation sites.

Defects of neuromuscular junction transmission (eg, myasthenia gravis, Lambert-Eaton myasthenic syndrome) exhibit changes in motor response amplitudes during a volley of stimuli when tested with repetitive nerve stimulation.

\section{NEEDLE ELECTRODE EXAMINATION}

Needle electrode examination (Figure 3) involves inserting a needle into a muscle to record spontaneous and volitional electrical activity generated within muscle fibers during rest and active muscle contraction.

The test is typically performed on multiple muscles: between 6 (for a single-limb study) and 15 muscles (for a multiple-limb study). An electrode inserted in the muscle belly records electrical activity in the muscle at rest
A motor nerve, composed of numerous axons (represented by a single neuron), is stimulated through the skin with a pulse of current administered through a stimulator, with enough current to depolarize all of the nerve's axons. Recording electrodes on the surface of the skin overlying the innervated muscle (not pictured) produce a tracing of electric potential over time, which represents the depolarization of all activated muscle cells.

This deflection from the electric baseline is called the compound muscle action potential (CMAP). The time between stimulation at a distal site and the initial deflection of the CMAP is called the distal latency (DL), which is determined by the size and myelination of the motor nerve, as well as transmission across the neuromuscular junction and within the muscle itself. Motor nerves are often stimulated proximally as well, which allows for calculation of a conduction time and associated conduction velocity across a segment of the nerve. This parameter does not include the neuromuscular junction or intramuscular transmission, and represents purely nerve conduction within a nerve segment.

and during voluntary contraction to assess the integrity of the nerve-muscle connection and the presence of muscle disease.

At rest. Abnormal spontaneous activity in the form of fibrillation or positive sharp wave potentials signifies loss of muscle innervation, necrosis, or inflammation (Table 1).

During voluntary muscle activation. The needle electrode records the size, morphology, and firing pattern of a motor unit action potential (ie, an electrical discharge composed of the individual muscle fiber action potentials generated by activation of a single motor neuron in the spinal cord). The pattern of firing in relation to increasing effort is called the recruitment pattern (Table 2).

\section{INTERPRETING RESULTS}

Nerve conduction studies and needle electrode examination can help address the following questions:

Where is the lesion? Is it in the nerve root, plexus, peripheral nerve, neuromuscular junction, or muscle?

What is the pathophysiologic nature of the disorder? If neuropathy, is it due to demyelination or to axon loss? If myopathy, is it due to inflammation and necrosis?

What is the chronicity of the problem? 


\section{GINSBERG AND COLLEAGUES}

\section{Nerve conduction studies: Abnormal patterns}
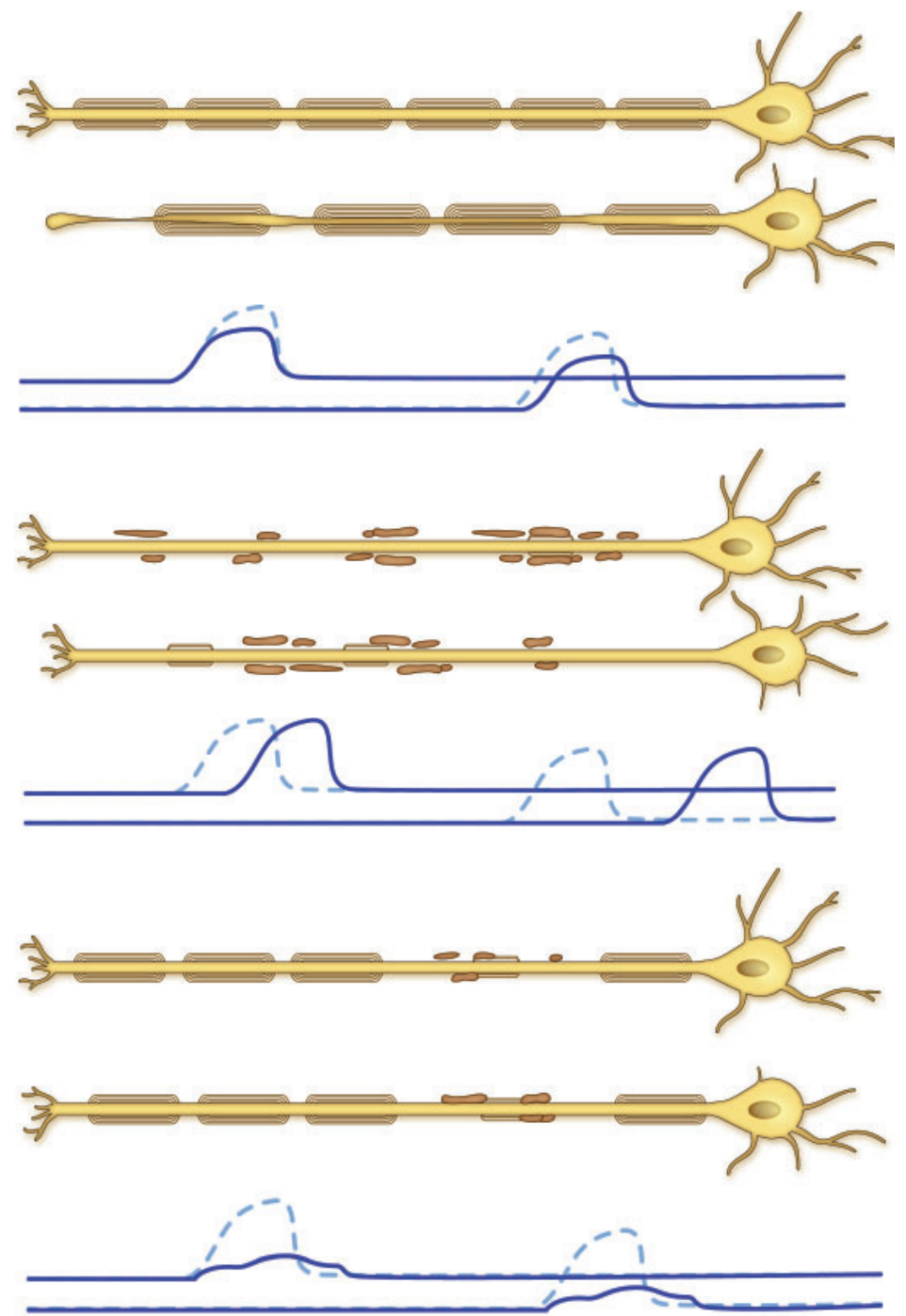

Figure 2.

Is it acute, subacute, chronic, or chronic with ongoing denervation?

What is the electrical severity of the problem?

Electrodiagnostic testing can also reveal specific clues to etiology, such as myotonia in a patient with suspected myopathy.

\section{LIMITATIONS OF ELECTRODIAGNOSIS}

Electrodiagnosis has limitations.

\section{It does not evaluate small fibers}

Nerve conduction studies assess the integrity of only large-diameter axons. Small-diameter
Axon loss. When axons are lost, there are fewer excitable axons, and therefore fewer muscle cells are excited, resulting in a lower compound muscle action potential (CMAP) regardless of stimulation site. This can occur in peripheral neuropathy or motor neuron disease. The dashed tracings represent normal, solid tracings are abnormal.

Diffuse demyelination. For diffusely disrupted myelin, distal latency is prolonged, and the conduction velocity is slow, but the CMAP retains normal amplitude because all of the axons are still available to depolarize the same number of muscle cells. This may be seen in hereditary demyelinating neuropathies, such as CharcotMarie-Tooth disease.

Focal demyelination. For focal demyelination over a portion of nerve, focal slowing occurs only over the affected segments. In addition, the conduction in some neurons is too slow to cross the area of focal demyelination. This is called conduction block, and results in a more than $50 \%$ reduction of CMAP amplitude when stimulating proximal to the lesion. Because focal demyelination typically affects different neurons to varying degrees, the action potentials arrive at the muscle at more variable times, leading to a "spreading" of the CMAP, known as temporal dispersion. This pattern can be seen in some types of compressive nerve injury (eg, ulnar neuropathy at the elbow) and diffuse acquired demyelinating polyneuropathies (eg, Guillain-Barré syndrome). fibers that predominantly comprise the autonomic, temperature-sensing, and pain-sensing portions of the peripheral nervous system generate electrical fields too small to be recorded with routine laboratory techniques. Hence, patients with small-fiber sensory neuropathy and those with radiculopathy only manifested by pain (affecting only sensory root fibers and not motor root fibers) will likely have normal results.

\section{It gives clues, but not a specific diagnosis}

Electrodiagnostic testing helps locate problems and objectively measures a portion of the peripheral nervous and neuromuscular sys- 


\section{Needle electrode examination in normal and diseased muscle}

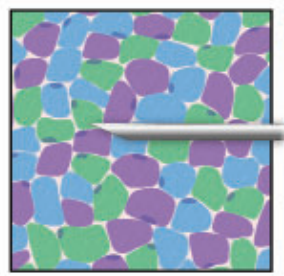

Normal

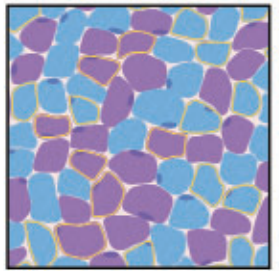

Neurogenic

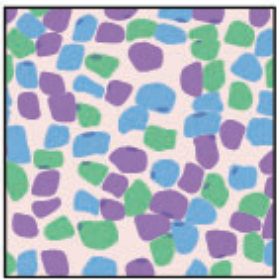

Myopathic
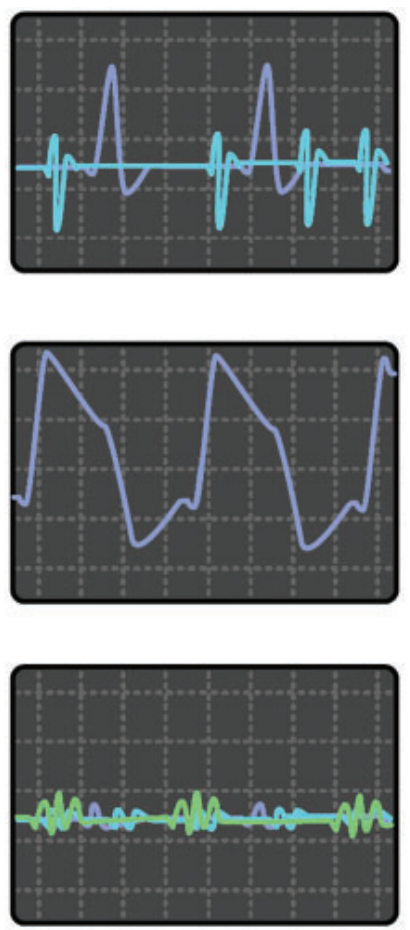

Normal. The recording needle is shown inserted into muscle perpendicular to the long axis of the muscle fibers. The electrode captures activity within a small range surrounding the needle tip. Normal tissue contains a mixture of different motor units (single units denoted by color). When a patient activates the muscle through voluntary control, force is generated by the orderly recruitment of additional motor units and an increase in the firing rate of motor units. The firing motor units are visualized to the right as tracings (color coding not present on actual reading). Each motor unit has a distinct morphology.

In neurogenic conditions, motor units are lost (represented by loss of the green motor unit), but if nearby motor units are intact, they can reinnervate the muscle fibers that have lost innervation (represented by increase of blue and purple-coded muscle fibers). During electrical activation, fewer motor units are available to generate the same level of force, so the remaining units must fire at a higher frequency ("reduced recruitment"). The size of the motor unit is increased because more muscle fibers now belong to each motor unit due to reinnervation.

In myopathic conditions, muscle fibers become smaller, although the motor units remain intact. In order to generate the same level of force, more motor units need to be activated ("early recruitment"). Motor units appear small due to electrical potentials generated from the smaller muscle fibers.

Figure 3.

tems. It does not usually identify the specific underlying cause of a condition and is best viewed as an extension of the physical examination. However, it often plays an important role in defining the differential diagnosis and directing further laboratory and imaging tests.

\section{It is less useful for elderly patients}

Nerve conduction studies are less reliable in advanced age. For example, sensory responses are not obtainable in the lower limbs of many healthy adults over age 75 , making electrodiagnostic testing less useful for diagnosing polyneuropathy. ${ }^{1}$

\section{It does not reveal much about the central nervous system}

Electrodiagnostic testing does not adequately assess the central nervous system. It may demonstrate nonspecific abnormalities in central nervous system disorders, but findings cannot be used to definitively locate or diagnose a central nervous system lesion. Electrodiagnostic testing may be technically limited by central disorders of motor unit control.

\section{It may require mild sedation}

Although most patients tolerate electrodiagnostic testing well, those with especially low pain tolerance or lacking understanding of the testing (eg, children) may require premedication.

\section{Some heart devices rule it out}

In general, electrodiagnostic testing is safe. However, nerve conductions studies should not be performed near catheters and electrodes that directly reach the heart (eg, pacemakers with external leads, catheters measuring intracardiac pressures), although having an internalized pacemaker or defibrillator is not a contraindication. ${ }^{2}$

\section{Risks of infection, bleeding}

Needle electrode examination carries a small risk of infection and bleeding. Laboratories differ in their approach for patients on anticoagulation therapy. In general, even with anticoagulation, the risk of clinically significant bleeding is low, and risk associated with discontinuing anticoagulation therapy should be balanced against this risk. ${ }^{3}$ For patients undergoing electrodiagnosis who stay on anticoagulation, the 
TABLE 1

\section{Commonly observed or notable abnormal spontaneous activity}

\begin{tabular}{|c|c|c|}
\hline Term & Description & Clinical significance \\
\hline \multirow[t]{2}{*}{$\begin{array}{l}\text { Fibrillation potentials } \\
\text { and positive sharp } \\
\text { waves }\end{array}$} & \multirow{2}{*}{$\begin{array}{l}\text { Spontaneous muscle fiber } \\
\text { potentials recorded during rest; } \\
\text { morphology and firing regularity } \\
\text { determine categorization as fibril- } \\
\text { lation potentials or positive sharp } \\
\text { waves }\end{array}$} & $\begin{array}{l}\text { Muscle fibers are remaining without inner- } \\
\text { vation, generally a sign of recent or ongoing } \\
\text { denervation in neurogenic conditions }\end{array}$ \\
\hline & & $\begin{array}{l}\text { In myopathic conditions, they may indicate } \\
\text { inflammatory or necrotizing myopathies }\end{array}$ \\
\hline $\begin{array}{l}\text { Fasciculation } \\
\text { potentials }\end{array}$ & $\begin{array}{l}\text { Spontaneous, irregularly firing } \\
\text { motor unit discharges }\end{array}$ & $\begin{array}{l}\text { May be seen occasionally in chronic neuro- } \\
\text { genic conditions of any kind, but are seen } \\
\text { more diffusely in disorders of the anterior } \\
\text { horn cell and motor neuron disease }\end{array}$ \\
\hline \multirow[t]{2}{*}{ Myotonic discharges } & \multirow{2}{*}{$\begin{array}{l}\text { Single muscle fiber firing } \\
\text { repetitively in a waxing and } \\
\text { waning pattern at high frequency }\end{array}$} & $\begin{array}{l}\text { When diffuse and prominent, indicates a } \\
\text { myotonic disorder }\end{array}$ \\
\hline & & $\begin{array}{l}\text { Can also rarely be seen in any chronic } \\
\text { neurogenic or myopathic condition }\end{array}$ \\
\hline $\begin{array}{l}\text { Complex repetitive } \\
\text { discharges }\end{array}$ & $\begin{array}{l}\text { Time-locked repetitive firing of a } \\
\text { group of muscle fibers, with sud- } \\
\text { den start and stop of bursts }\end{array}$ & $\begin{array}{l}\text { Very chronic neurogenic or myopathic } \\
\text { conditions }\end{array}$ \\
\hline $\begin{array}{l}\text { Neuromyotonic } \\
\text { discharges }\end{array}$ & $\begin{array}{l}\text { Single motor unit firing repetitively } \\
\text { at a very high frequency }\end{array}$ & $\begin{array}{l}\text { Typically, disorders of voltage-gated } \\
\text { potassium channels }\end{array}$ \\
\hline Myokymic discharges & $\begin{array}{l}\text { Single motor unit firing in regularly } \\
\text { recurring bursts }\end{array}$ & $\begin{array}{l}\text { Most commonly associated with chronic } \\
\text { demyelination and radiation plexopathy }\end{array}$ \\
\hline
\end{tabular}

needle electrode examination may be tailored to exclude particularly vulnerable sites.

Examination of certain muscles (especially the diaphragm, rhomboid major, and serratus anterior) entails a higher risk of pneumothorax.

\section{SPECIFIC INDICATIONS}

In general, electrodiagnostic testing adds value to the diagnostic workup of many common symptoms and conditions by suggesting previously unsuspected diagnoses and further diagnostic tests or treatments. 4,5

\section{FOCAL SENSORY AND MOTOR SYMPTOMS}

Patients with many conditions presenting with focal sensory and motor symptoms can benefit from electrodiagnostic testing.

Acute traumatic nerve injury

Peripheral nerves may be injured by blunt or penetrating trauma, stretch injury, and secondary ischemia (eg, from compartment syndrome). Electrodiagnosis can assess nerve continuity, injury severity, and prognosis, which may be especially helpful if peripheral nerve surgery is being considered.

Nerve conduction studies may be useful during the acute phase of an injury (within the first 24-72 hours) if nerve trunk stimulation can be performed above and below the lesion site to assess for conduction block or discontinuity. A repeat study at least 10 days after the injury is usually necessary to assess for maximal deterioration of sensory and motor responses, at which time wallerian degeneration should be complete, and a response from distal stimulation will be absent with complete axonal injuries. ${ }^{6}$

However, needle electrode evaluation is not usually useful until 3 weeks after an injury, when active denervation features may become apparent, so if a single study is requested, it should be done 3 weeks after the onset of neurologic deficits.

\section{Electrodiag- nosis often helps define the differential diagnosis and directs further evaluation}


TABLE 2

\section{Glossary of common electrodiagnostic terms}

Term Description Clinical pearls

\begin{tabular}{|c|c|c|}
\hline Chronic denervation & $\begin{array}{l}\text { Remote axon loss identified by long-duration, } \\
\text { high-amplitude motor units firing with a } \\
\text { reduced recruitment pattern }\end{array}$ & $\begin{array}{l}\text { Generally denotes a process that started at least } \\
\text { several months before the examination }\end{array}$ \\
\hline $\begin{array}{l}\text { Active or ongoing } \\
\text { denervation }\end{array}$ & $\begin{array}{l}\text { A muscle exhibiting positive sharp waves or } \\
\text { fibrillation potentials, reflecting a subacute } \\
\text { (or more long-standing but uncompensated) } \\
\text { neurogenic or axon-loss process }\end{array}$ & $\begin{array}{l}\text { Does not always imply a truly active process. Fi- } \\
\text { brillation potentials and positive sharp waves are } \\
\text { observed whenever a muscle fiber is awaiting } \\
\text { reinnervation. These findings generally appear } \\
\text { by about } 3 \text { weeks after the onset of injury and } \\
\text { resolve within a few months, but may persist for } \\
\text { longer in distal muscles and when reinnervation } \\
\text { mechanisms are not fully successful or complete }\end{array}$ \\
\hline
\end{tabular}

\begin{tabular}{|c|c|c|}
\hline $\begin{array}{l}\text { Intraspinal canal } \\
\text { lesion or process }\end{array}$ & $\begin{array}{l}\text { Electrodiagnostic testing characterized by } \\
\text { neurogenic or axon-loss changes in muscles } \\
\text { of } 1 \text { or more specific myotomes (eg, a spinal } \\
\text { root or segment derivative) without sensory } \\
\text { findings }\end{array}$ & $\begin{array}{l}\text { The lesion is proximal to the dorsal root gan- } \\
\text { glion. Most of these lesions are compressive } \\
\text { radiculopathies; but infrequently; other lesions } \\
\text { such as motor neuron disease produce similar } \\
\text { findings }\end{array}$ \\
\hline Neurogenic & $\begin{array}{l}\text { Electrodiagnostic features resulting from } \\
\text { lesions of the anterior horn cell, nerve root, } \\
\text { plexus, or nerve }\end{array}$ & $\begin{array}{l}\text { Neurogenic findings are further refined by distri- } \\
\text { bution and the presence or absence of sensory } \\
\text { findings }\end{array}$ \\
\hline Myopathic & $\begin{array}{l}\text { Electrodiagnostic features of muscle disease, } \\
\text { including low amplitude, short duration, and } \\
\text { polyphasic motor units }\end{array}$ & $\begin{array}{l}\text { Electrodiagnostic testing may be less sensitive } \\
\text { in many myopathies than in neurogenic condi- } \\
\text { tions }\end{array}$ \\
\hline Irritable myopathy & $\begin{array}{l}\text { When myopathic features are accompanied } \\
\text { by diffuse fibrillation potentials, positive sharp } \\
\text { waves, or both }\end{array}$ & $\begin{array}{l}\text { Suggestive of inflammatory or necrotizing } \\
\text { etiologies, but not pathognomonic }\end{array}$ \\
\hline Motor unit instability & $\begin{array}{l}\text { The same motor unit on needle electrode } \\
\text { examination varies in morphology from one } \\
\text { firing to the next }\end{array}$ & $\begin{array}{l}\text { Indicates dysfunction at the neuromuscular } \\
\text { junction, but it can be seen in neurogenic condi- } \\
\text { tions during early reinnervation, when neuro- } \\
\text { muscular junctions are immature }\end{array}$ \\
\hline Reduced activation & $\begin{array}{l}\text { Suboptimal voluntary activation of a muscle } \\
\text { resulting from central nervous system causes }\end{array}$ & $\begin{array}{l}\text { Can result from pain, cognitive dysfunction, poor } \\
\text { effort, or upper motor neuron pathology, and } \\
\text { indicates that the data may be of lower yield }\end{array}$ \\
\hline \multirow[t]{2}{*}{ Conduction block } & \multirow{2}{*}{$\begin{array}{l}\text { Motor response in a nerve conduction study } \\
\text { has }>50 \% \text { reduced response when stimulating } \\
\text { at a more proximal location }\end{array}$} & Indicates focal demyelination \\
\hline & & $\begin{array}{l}\text { When occurring at noncompression sites or in } \\
\text { multiple nerves, can suggest acquired demyelin- } \\
\text { ating polyneuropathies }\end{array}$ \\
\hline
\end{tabular}

\section{Carpal tunnel syndrome}

Carpal tunnel syndrome is one of the most common peripheral nerve disorders and can cause significant pain and dysfunction. ${ }^{7-9}$ When typical symptoms and signs are present, the diagnosis may be straightforward. How- ever, in other cases, the sensory distribution of pain and paresthesias lie outside of the classic median nerve distribution, and in addition, other conditions can mimic it.

Electrodiagnosis is most applicable for evaluating suspected carpal tunnel syndrome 
when the diagnosis is uncertain, when initial conservative therapy has been unsuccessful, or when surgery is being considered. Specifically, electrodiagnostic testing can help with the following:

Establishing a diagnosis. Diagnostic accuracy is high, especially when using specialized nerve conduction techniques (eg, palmar mixed nerve studies), with sensitivities of about $85 \%$ and specificities around $97 \% .{ }^{9}$ Standard electrodiagnosis may also exclude other neuromuscular diagnoses, such as cervical radiculopathy, other upper limb mononeuropathies, and brachial plexopathy.

Evaluating need for surgery. Electrodiagnostic testing may help determine indications for surgical release of a trapped median nerve. Lesions that are electrically moderate may be associated with a better prognosis, presumably because normal studies predict a disorder other than carpal tunnel syndrome, and severe findings suggest irreversible axon loss. ${ }^{10}$

Postoperative assessment. Electrodiagnostic testing is sometimes used after surgery if the outcomes are suboptimal. Electrodiagnostic findings typically improve after surgery, but abnormalities occasionally persist even after symptoms improve.

Neuromuscular ultrasonography. Interest has been growing for evaluating carpal tunnel syndrome with neuromuscular ultrasonography, as it has demonstrated favorable diagnostic accuracy. ${ }^{11}$ However, it provides information that is complementary to electrodiagnostic testing results and is not useful for assessing severity. Neuromuscular ultrasonography should be considered for patients who prefer not to undergo electrodiagnostic testing or may not tolerate it. It may also be used to assess other structural causes of carpal tunnel syndrome in unusual presentations, or to aid in surgical planning or postoperative evaluation.

\section{Ulnar neuropathy at the elbow}

Ulnar neuropathy at the elbow is only slightly less common than carpal tunnel syndrome. ${ }^{12}$ Typical symptoms are numbness or pain in the hand, with or without weakness and atrophy of ulnar-innervated muscles. The differential diagnosis often includes C8 radiculopathy, lower trunk brachial plexus lesions, muscu- loskeletal conditions, and ulnar nerve lesions located elsewhere (eg, at the wrist).

Electrodiagnostic testing can be useful for diagnosing ulnar neuropathy at the elbow, and guidelines have been published on electrodiagnostic techniques and criteria. ${ }^{13}$ However, several challenges are unique to this condition. The anatomy of the nerve, variation in lesion site in the region of the elbow, and sparing of the muscles of the forearm that are innervated by the ulnar nerve, even with clear lesions at the elbow, can make electrical localization difficult, especially if the lesion primarily involves axon loss.

Diagnostic criteria may also have substantially different accuracies depending on the pretest probability of an ulnar neuropathy at the elbow. ${ }^{14}$ If an ulnar neuropathy is nonlocalizable by nerve conduction studies, alternative diagnostic techniques (eg, neuromuscular ultrasonography) should be considered to aid in localization, ${ }^{15}$ especially for a moderate or severe lesion that is being considered for surgery.

Electrodiagnosis may also help with prognostic guidance. Conduction block at the elbow indicates that focal demyelination may be contributing substantially to symptoms, which is associated with a more favorable recovery. ${ }^{16}$

\section{Radiculopathy}

Patients are commonly referred for electrodiagnostic testing to evaluate radiculopathies. Electrodiagnosis can typically identify the root level, chronicity, and electrical severity of a radiculopathy. Several conditions and settings merit special consideration, as follows:

Intraspinal compressive radiculopathies. These are usually located proximal to the dorsal root ganglion, so sensory nerve conduction studies are typically normal despite significant symptoms of pain and numbness. Motor nerve conduction studies often show only minimal axon loss because most lesions cause damage to a minority of nerve fibers. Needle electrode examination is often the most useful, as it reveals motor axon loss (neurogenic) changes in a myotomal pattern. Diagnosis typically requires examination of multiple muscles to isolate the affected level due to some interindividual variation and overlapping root innervation in many muscles. ${ }^{17}$
Electrodiagnosis may especially be helpful if peripheral nerve surgery is being considered 
Demyelinating conditions. For predominantly demyelinating diseases, the only changes on electrodiagnostic testing are in the recruitment pattern of motor unit action potentials in affected muscles, which may be subtle.

Predominant sensory involvement. Radiculopathies that mainly affect sensory fibers do not result in significantly abnormal findings on electrodiagnostic testing.

Anatomic considerations. Certain radiculopathies may be difficult to isolate to a single level (eg, differentiating between C8 and T1, and $\mathrm{C} 6$ and $\mathrm{C} 7$ radiculopathies). In addition, electrodiagnostic testing does not truly localize a lesion to the nerve root in the intervertebral foramen, but rather proximal to the dorsal root ganglion. This means that electrodiagnosis cannot differentiate between a root and anterior horn cell lesion within the spinal cord.

The overall sensitivity and specificity of electrodiagnostic testing for radiculopathy is difficult to determine, with reported values varying widely. ${ }^{18}$ This is partly due to lack of a gold standard and the various combinations of criteria that can be used for diagnosis. In general, electrodiagnosis can be used to determine if a radicular lesion (eg, one identified on magnetic resonance imaging) is severe enough to have caused motor axon loss and whether the lesion is acute, chronic and healed, or chronic and unhealed (ie, chronic with significant active and ongoing denervation).

in diagnosing amyotrophic lateral sclerosis

\section{GENERALIZED SENSORY AND MOTOR SYMPTOMS}

Other conditions that can be evaluated with electrodiagnosis are characterized by a more generalized presentation.

\section{Polyneuropathy}

Distal, symmetric axon-loss ("axonal") polyneuropathy is a common condition that may affect large-fiber or small-fiber nerves, or both.

Evidence is conflicting regarding the value of electrodiagnostic testing for assessing suspected polyneuropathy., ${ }^{3,19-21}$ Some experts argue that it does not add substantial benefit, as it rarely yields a specific underlying cause, and results do not affect treatment. ${ }^{22}$ However, electrodiagnostic testing can identify alternative or concomitant neuromuscular di- agnoses, such as radiculopathy or mononeuropathies (eg, carpal tunnel syndrome). It can also distinguish demyelinating polyneuropathies (characterized by slowing of conduction velocities, prolonged distal latencies, conduction blocks, dispersion of the motor response waveform, and prolonged late responses) from axon-loss polyneuropathies, which do not significantly exhibit these features but will display reduced response amplitudes. This has important management ramifications, as demyelinating polyneuropathies and polyradiculoneuropathies are often associated with inflammatory conditions and respond to specific treatments.

Axon-loss polyneuropathy is considerably more common than demyelinating polyneuropathies. Diabetes mellitus confers high risk for axon-loss polyneuropathy ${ }^{23}$ but is also associated with increased risk for other neuropathic disorders, including carpal tunnel syndrome, ulnar neuropathy, and diabetic radiculoplexus neuropathy (also known as diabetic amyotrophy).

Electrodiagnostic testing should be considered for polyneuropathy in the evaluation of patients with prominent weakness or gait abnormality, asymmetrical patterns, early upper extremity involvement, rapid progression, and diffuse loss of reflexes.

\section{Limitations of electrodiagnosis for assessing polyneuropathy}

Referring physicians should be aware of the following limitations of electrodiagnosis for assessing polyneuropathy:

It is less useful for small-fiber dysfunction. Patients whose history and examination indicate only small-fiber dysfunction are likely to have normal study results and may benefit more from alternative evaluations, such as skin biopsy for intraepidermal nerve fiber density measurement and the QSART (quantitative sudomotor axon reflex test) to assess for small-fiber neuropathy.

It is less useful for elderly patients with mild symptoms. Differentiating between normal age-related loss of sensory responses and features of polyneuropathy may be difficult.

Incidental findings may not be relevant. Especially in older patients, incidental electrodiagnostic findings (eg, an old radiculopathy, 
carpal tunnel syndrome) may not help elucidate the cause of symptoms. Electrodiagnostic findings must always be evaluated in the context of a patient's target clinical features.

\section{Demyelinating polyneuropathy}

Electrodiagnostic testing plays an important role in diagnosing demyelinating polyneuropathies, which have substantially different management implications than axon-loss polyneuropathies. Electrodiagnostic testing can determine the likelihood that a demyelinating polyneuropathy is hereditary or acquired, the types of nerves affected, and the degree of concomitant axon loss. However, skill is required for acquiring and interpreting the electrodiagnostic data, because mild or focal demyelinating-type findings may actually be due to axon-loss polyneuropathy or compressive etiologies. The European Federation of Neurological Societies and the Peripheral Nerve Society have published guidelines for accurate electrodiagnosis, but misdiagnosis of chronic inflammatory demyelinating polyneuropathy commonly occurs and may lead to unnecessary and potentially harmful therapy. ${ }^{24,25}$

\section{Generalized weakness}

Weakness has diverse causes. A first approximation is often made clinically, differentiating upper from lower motor neuron-type weakness. Those with lower motor neuron-type weakness may have lesions at the level of the anterior horn cell, nerve root, plexus, peripheral nerve, neuromuscular junction, muscle, or some combination of these sites.

Electrodiagnostic testing can be a useful adjunct to a physical examination to help refine localization in the peripheral nervous system (including neuromuscular junction and muscle). In a prospective study of patients presenting with weakness, electrodiagnosis identified a single cause in approximately $80 \%$ of patients, with about $30 \%$ of diagnoses unsuspected before testing. ${ }^{26}$

Central disorders of motor control including upper motor neuron disorders may show a pattern of reduced voluntary activation on needle electrode examination. This finding, when pronounced, can suggest upper motor neuron localization. However, it is not specific and can also be seen in studies confounded by pain or lack of voluntary effort.

\section{Motor neuron disease}

Electrodiagnostic testing plays an important role in diagnosing motor neuron diseases, most commonly amyotrophic lateral sclerosis (ALS), a degenerative disorder of the upper and lower motor neurons. Diagnosis relies on clinical demonstration of progressive combined upper and lower motor neuron signs without alternative explanation, but electrodiagnosis can identify denervation that may not be apparent clinically.

Several sets of diagnostic criteria are available for ALS, the two most common being the the Awaji criteria and the revised El Escorial criteria. ${ }^{27,28}$ The Awaji criteria have better sensitivity for diagnosing ALS, although possibly not for all patients. ${ }^{29,30}$

Motor neuron disease requires extensive electrodiagnostic evaluation. Nerve conduction studies should be performed to exclude polyneuropathy. Needle electrode examination includes study of the upper and lower extremities, thoracic paraspinal muscles, and often, cranial nerve-supplied muscles. A tiered approach may minimize the number of muscles requiring examination. ${ }^{31}$

Key features suggesting a diagnosis of motor neuron disease are the following:

- Chronic and active motor axon loss in muscles from multiple myotomes and peripheral nerve distributions within each of at least 3 body regions

- Progressive clinical features of upper and lower motor neuron deficits

- Fasciculations on needle electrode examination and clinical inspection. Although they may be seen in other neurogenic conditions and in healthy people, when seen in association with weakness, atrophy, and chronic denervation features, they qualify by the Awaji criteria as a surrogate for active denervation in a muscle.

Electrodiagnostic testing is also useful in identifying ALS mimics, including multifocal motor neuropathy with conduction block, myopathies, neuromuscular junction disorders, structural radiculopathies, and severe neuropathies. Other motor neuron diseases include spinobulbar muscular atrophy (Kennedy disease) and spinal muscular atrophy.

\section{Myopathies comprise a broad spectrum of generalized disorders that primarily affect skeletal muscles}




\section{Needle electrode examination: Spontaneous activity}

Insertional/spontaneous

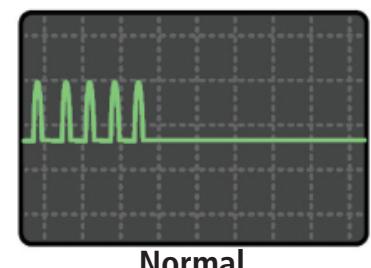

Normal

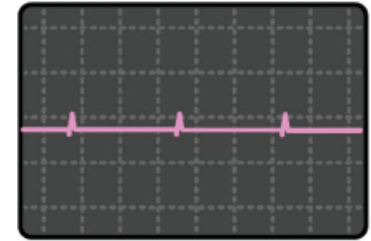

Fibrillation

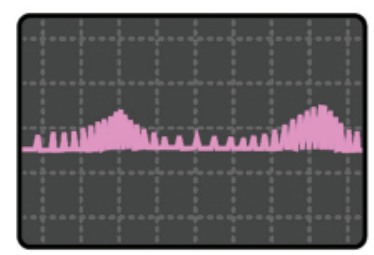

Myotonia

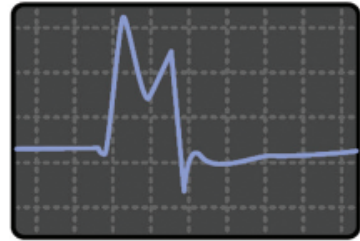

Fasciculation

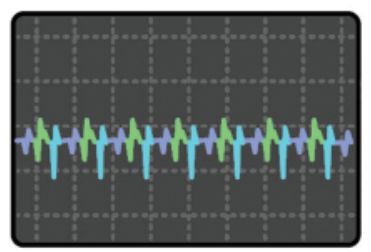

Complex repetitive discharge

Normal. Movement of the needle through uncontracted (relaxed) muscle causes irritation of muscle fiber membranes and a brief burst of muscle fiber depolarizations.

Abnormal. Most other spontaneous activity is abnormal. Activity is categorized by source of the discharge (ie, muscle fiber, motor unit, or muscle fiber circuit/nonmotor unit chain of fibers), the firing pattern (ie, regular, irregular, semiregular), and frequency. Most spontaneous activity is not specific to myopathic or neurogenic conditions, but may yield information about chronicity or underlying etiology. See Table 1 for detailed descriptions of abnormal spontaneous activity.

Figure 4.

Electrodiag-
nosis can help
narrow
the differential
diagnosis
based on the
distribution
of muscle
involvement

Myopathy

Myopathies comprise a broad spectrum of generalized disorders that primarily affect skeletal muscles. Nerve conduction studies are typically normal in most myopathies because sensory functions are unaffected and the muscles that are routinely tested are distal and less likely to be affected by a myopathy.

Needle electrode examination is more valuable, revealing myopathic motor units (short duration, low amplitude, polyphasic morphology). ${ }^{32}$ However, myopathies that predominantly affect type II muscle fibers (notably, corticosteroid-induced myopathy) may have normal results on needle electrode examination, as these fibers are not typically evaluated. ${ }^{6}$

The absence of fibrillation potentials has a negative predictive value of about $80 \%$ to $90 \%$ for inflammation, necrosis, fiber splitting, or vacuolar changes on muscle biopsy. This information may be helpful in deciding which patients warrant a biopsy. ${ }^{33}$

Electrodiagnosis can help diagnose some myopathies and also perform the following valuable functions:

- Exclude neurogenic and neuromuscular junction etiologies that may mimic myopathies (eg, motor neuron disease, myasthenia gravis)

- Identify unusual myopathic needle electrode examination patterns (eg, myotonia)

- Narrow the differential diagnosis based on the distribution of muscle involvement (eg, inclusion body myositis).

In addition, needle electrode examination features may suggest (but not distinguish between) the following causes of myopathy in the appropriate clinical context:

- Necrosis (eg, anti-signal recognition particle and anti-hydroxy-3-methylglutarylCoA reductase autoantibody-related myopathies)

- Inflammation (eg, polymyositis and dermatomyositis).

"Irritative" features (ie, fibrillation or positive sharp wave potentials) in conjunction with motor unit potential configurational and recruitment changes consistent with myopa- 
thy may occur in both types of myopathy. Differentiating between them depends primarily on histopathology (ie, necrotizing myopathy predominantly has features of myofiber degeneration without the inflammatory infiltrates typical of an inflammatory myopathy).

Myotonia is a unique electrical phenomenon (Figure 4, Table 1 ) resulting from quantitative or qualitative dysfunction of sodium and chloride channels in the muscle cell membrane. Although it may occur secondary to a wide variety of neuromuscular pathologies, prominent or diffuse myotonia is associated with a relatively small differential diagnosis (including myotonic dystrophies, inherited sodium and chloride channelopathies, and Pompe disease).

Needle electrode examination can also help identify an affected muscle for biopsy. However, the biopsied muscle is typically chosen from the contralateral side to avoid needle track artifacts. ${ }^{34}$

\section{Myasthenia gravis}

Electrodiagnosis can play an important role in evaluating patients with suspected disorders of neuromuscular junction transmission. The most common such disorder is autoimmune myasthenia gravis, which is diagnosed clinically but supported by ancillary testing. Electrodiagnosis is not always necessary if the history and autoantibody profile are consistent with the diagnosis, but it can be useful in cases in which antibody testing is negative and the diagnosis is unclear. It may also play a role in determining whether subjective weakness in a patient with myasthenia gravis is caused by uncontrolled disease or other causes.

In postsynaptic neuromuscular junction disorders such as myasthenia gravis, slow repetitive stimulation at 2 to $5 \mathrm{~Hz}$ produces a stereotyped, progressive decrease in the re-

\section{REFERENCES}

1. Tavee JO, Polston D, Zhou L, Shields RW, Butler RS, Levin KH. Sural sensory nerve action potential, epidermal nerve fiber density, and quantitative sudomotor axon reflex in the healthy elderly. Muscle Nerve 2014; 49(4):564-569. doi:10.1002/mus.23971

2. Cronin EM, Gray J, Abi-Saleh B, Wilkoff BL, Levin KH. Safety of repetitive nerve stimulation in patients with cardiac implantable electronic devices. Muscle Nerve 2013; 47(6):840-844. doi:10.1002/mus.23707

3. Kothari MJ, Blakeslee MA, Reichwein R, Simmons Z, Logigian EL. Electrodiagnostic studies: are they useful in clinical practice? Arch Phys Med Rehabil 1998; 79(12):1510-1511. doi:10.1016/s0003-9993(98)90411-7 corded motor response amplitude or area in weak muscles.

The overall accuracy of the test is dependent on the muscle studied, the reference values used, and type of myasthenia gravis (ie, generalized or oculobulbar, the latter of which does not significantly involve limb muscles). Sensitivities for repetitive nerve stimulation have been reported in the $40 \%$ to $50 \%$ range for generalized myasthenia gravis and in the $10 \%$ to $20 \%$ range for oculobulbar disease. ${ }^{35-37}$ Sensitivity might also be reduced if the patient has not appropriately discontinued pyridostigmine before testing. Specificity in facial muscles is reported close to $100 \%$. However, false-positives can occur from technical errors (which can be common in inexperienced hands) and disorders in which there is a secondary defect of neuromuscular junction transmission (eg, ALS). ${ }^{38}$ A negative test result cannot be used to exclude the diagnosis.

Needle electrode examination may reveal motor unit instability in disorders of neuromuscular junction transmission. When routine electrodiagnostic testing is nondiagnostic or when symptoms are not generalized, a singlefiber electromyographic study may be diagnostic. This technique is $90 \%$ to $100 \%$ sensitive for myasthenia gravis, but not as specific ${ }^{39}$; however, it requires significant patient cooperation and is technically demanding and time-consuming.

\section{BOTTOM LINE}

By keeping in mind the capabilities and limitations of electrodiagnosis, referring providers can obtain the greatest value from testing and provide reasonable expectations for patients. Results are optimized with testing by physicians trained in electrodiagnosis and interpreting the results in the context of a thorough history and physical examination.

4. Ginsberg MR, Morren JA. Utility of electrodiagnostic studies in patients referred with a diagnosis of polyneuropathy. Muscle Nerve 2020; 61(3):288-292. doi:10.1002/mus.26746

5. Bongers FJ, Schellevis FG, van den Bosch WJ, van der Zee J. Carpal tunnel syndrome in general practice (1987 and 2001): incidence and the role of occupational and non-occupational factors. Br J Gen Pract 2007; 57(534):36-39. pmid:17244422

6. Preston DC, Shapiro BE. Electromyography and Neuromuscular disorders: Clinical-Electrophysiologic Correlations. 3rd ed. Philadelphia, PA: Saunders; 2012.

7. Stevens JC, Sun S, Beard CM, O'Fallon WM, Kurland LT. Carpal tunnel syndrome in Rochester, Minnesota, 1961 to 1980. Neurology 1988; 38(1):134-138. doi:10.1212/wnl.38.1.134

8. Atroshi I, Gummesson C, Johnsson R, Ornstein E, Ranstam J, Rosén 
I. Prevalence of carpal tunnel syndrome in a general population. JAMA 1999; 282(2):153-158. doi:10.1001/jama.282.2.153

9. American Association of Electrodiagnostic Medicine, American Academy of Neurology, and American Academy of Physical Medicine and Rehabilitation. Practice parameter for electrodiagnostic studies in carpal tunnel syndrome: summary statement. Muscle Nerve 2002; 25(6):918-922. doi:10.1002/mus.10185

10. Bland JD. Do nerve conduction studies predict the outcome of carpal tunnel decompression? Muscle Nerve 2001; 24(7):935-940. doi:10.1002/mus.1091

11. Cartwright MS, Hobson-Webb LD, Boon AJ, et al. Evidence-based guideline: neuromuscular ultrasound for the diagnosis of carpal tunnel syndrome. Muscle Nerve 2012; 46(2):287-293. doi:10.1002/mus.23389

12. An TW, Evanoff BA, Boyer MI, Osei DA. The prevalence of cubital tunnel syndrome: a cross-sectional study in a U.S. metropolitan cohort. J Bone Joint Surg Am 2017; 99(5):408-416. doi:10.2106/JBJS.15.01162

13. Practice parameter for electrodiagnostic studies in ulnar neuropathy at the elbow: summary statement. American Association of Electrodiagnostic Medicine, American Academy of Neurology, American Academy of Physical Medicine and Rehabilitation. Muscle Nerve 1999; 22(3):408-411. doi:10.1002/(sici)1097-4598(199903)22:3<408::aid-mus16>3.0.co;2-7

14. Logigian EL, Villanueva R, Twydell PT, et al. Electrodiagnosis of ulnar neuropathy at the elbow (UNE): a Bayesian approach. Muscle Nerve 2014; 49(3):337-344. doi:10.1002/mus.23913

15. Walker FO, Cartwright MS, Wiesler ER, Caress J. Ultrasound of nerve and muscle. Clin Neurophysiol 2004; 115(3):495-507. doi:10.1016/j.clinph.2003.10.022

16. Beekman R, Zijlstra W, Visser LH. A novel points system to predict the prognosis of ulnar neuropathy at the elbow. Muscle Nerve 2017; 55(5):698-705. doi:10.1002/mus.25406

17. Tsao BE, Levin KH, Bodner RA. Comparison of surgical and electrodiagnostic findings in single root lumbosacral radiculopathies. Muscle Nerve 2003; 27(1):60-64. doi:10.1002/mus.10291

18. Cho SC, Ferrante MA, Levin KH, Harmon RL, So YT. Utility of electrodiagnostic testing in evaluating patients with lumbosacral radiculopathy: an evidence-based review. Muscle Nerve 2010; 42(2):276-282. doi:10.1002/mus.21759

19. Cho SC, Siao-Tick-Chong P, So YT. Clinical utility of electrodiagnostic consultation in suspected polyneuropathy. Muscle Nerve 2004; 30(5):659-662. doi:10.1002/mus.20119

20. Bodofsky EB, Carter GT, England JD. Is electrodiagnostic testing for polyneuropathy overutilized? Muscle Nerve 2017; 55(3):301-304. doi:10.1002/mus.25464

21. Vrancken AF, Notermans NC, Wokke JH, Franssen $\mathbf{H}$. The realistic yield of lower leg SNAP amplitudes and SRAR in the routine evaluation of chronic axonal polyneuropathies. J Neurol 2008; 255(8):1127-1135. doi:10.1007/s00415-008-0817-7

22. Callaghan BC, Kerber KA, Lisabeth LL, et al. Role of neurologists and diagnostic tests on the management of distal symmetric polyneuropathy. JAMA Neurol 2014; 71(9):1143-1149. doi:10.1001/jamaneurol.2014.1279

23. Callaghan BC, Gao L, Li Y, et al. Diabetes and obesity are the main metabolic drivers of peripheral neuropathy. Ann Clin Transl Neurol 2018; 5(4):397-405. doi:10.1002/acn3.531

24. Allen JA, Ney J, Lewis RA. Electrodiagnostic errors contribute to chronic inflammatory demyelinating polyneuropathy misdiagnosis. Muscle Nerve 2018; 57(4):542-549. doi:10.1002/mus.25997

25. Van den Bergh PY, Hadden RD, Bouche P, et al. European Federa- tion of Neurological Societies/Peripheral Nerve Society guideline on management of chronic inflammatory demyelinating polyradiculoneuropathy: report of a joint task force of the European Federation of Neurological Societies and the Peripheral Nerve Society-first revision. Eur J Neurol 2010; 17(3):356-363. doi:10.1111/j.1468-1331.2009.02930.x

26. Nardin RA, Rutkove SB, Raynor EM. Diagnostic accuracy of electrodiagnostic testing in the evaluation of weakness. Muscle Nerve 2002; 26(2):201-205. doi:10.1002/mus.10192

27. de Carvalho $\mathbf{M}$, Dengler $\mathbf{R}$, Eisen $\mathbf{A}$, et al. Electrodiagnostic criteria for diagnosis of ALS. Clin Neurophysiol 2008; 119(3):497-503. doi:10.1016/j.clinph.2007.09.143

28. Brooks BR, Miller RG, Swash M, Munsat TL; World Federation of Neurology Research Group on Motor Neuron Diseases. El Escorial revisited: revised criteria for the diagnosis of amyotrophic lateral sclerosis. Amyotroph Lateral Scler Other Motor Neuron Disord 2000; 1(5):293-299. doi:10.1080/146608200300079536

29. Jang JS, Bae JS. AWAJI criteria are not always superior to the previous criteria: a meta-analysis. Muscle Nerve 2015; 51(6):822-829. doi:10.1002/mus.24575

30. Geevasinga N, Loy CT, Menon P, et al. Awaji criteria improves the diagnostic sensitivity in amyotrophic lateral sclerosis: a systematic review using individual patient data. Clin Neurophysiol 2016; 127(7):2684-2691. doi:10.1016/j.clinph.2016.04.005

31. Babu S, Pioro EP, Li J, Li Y. Optimizing muscle selection for electromyography in amyotrophic lateral sclerosis. Muscle Nerve 2017; 56(1):36-44. doi:10.1002/mus.25444

32. Rosow LK, Amato AA. The role of electrodiagnostic testing, imaging, and muscle biopsy in the investigation of muscle disease. Continuum (Minneap Minn) 2016; 22(6):1787-1802. doi:10.1212/01.CON.0000511068.61017.55

33. Sener U, Martinez-Thompson J, Laughlin RS, Dimberg EL, Rubin DI. Needle electromyography and histopathologic correlation in myopathies. Muscle Nerve 2019; 59(3):315-320. doi:10.1002/mus.26381

34. Engel WK. Focal myopathic changes produced by electromyographic and hypodermic needles. "Needle myopathy." Arch Neurol 1967; 16(5):509-511. doi:10.1001/archneur.1967.00470230061008

35. Zinman LH, O'Connor PW, Dadson KE, Leung RC, Ngo M, Bril V. Sensitivity of repetitive facial-nerve stimulation in patients with myasthenia gravis. Muscle Nerve 2006; 33(5):694-696. doi:10.1002/mus.20511

36. Abraham A, Alabdali M, Alsulaiman A, et al. Repetitive nerve stimulation cutoff values for the diagnosis of myasthenia gravis. Muscle Nerve 2017; 55(2):166-170. doi:10.1002/mus.25214

37. Chiou-Tan FY, Gilchrist JM. Repetitive nerve stimulation and singlefiber electromyography in the evaluation of patients with suspected myasthenia gravis or Lambert-Eaton myasthenic syndrome: review of recent literature. Muscle Nerve 2015; 52(3):455-462. doi:10.1002/mus.24745

38. Hatanaka Y, Higashihara M, Chiba T, Miyaji Y, Kawamura Y, Sonoo M. Utility of repetitive nerve stimulation test for ALS diagnosis. Clin Neurophysiol 2017; 128(5):823-829. doi:10.1016/j.clinph.2017.02.021

39. Padua L, Caliandro P, Di lasi G, Pazzaglia C, Ciaraffa F, Evoli A. Reliability of SFEMG in diagnosing myasthenia gravis: sensitivity and specificity calculated on 100 prospective cases. Clin Neurophysiol 2014; 125(6):1270-1273. doi:10.1016/j.clinph.2013.11.005

Address: Matthew R. Ginsberg, MD, Neurodevelopmental Science Center, Akron Children's Hospital, Considine Professional Building, 215 West Bowery St., Akron, OH 44308; MGinsberg@akronchildrens.org 